\title{
Avaliação do primeiro período clínico do trabalho de parto
}

\author{
Evaluation of the first clinical labor period \\ Evaluación del primer período clínico del trabajo de parto
}

Maria de Nazaré Jesus Oliveira ${ }^{1 *}$, Natalia Ferreira de Sousa ${ }^{2}$, Suelane dos Santos Silva ${ }^{2}$, Karla Joelma Bezerra Cunha ${ }^{3}$.

\section{RESUMO}

Objetivo: Elencar na literatura evidências cientifica acerca da importância da avaliação clínica no primeiro período do trabalho de parto. Metodologia: Trata-se de uma revisão integrativa da literatura, na qual consultou-se por meio de descritores a base BVS (Biblioteca Virtual em Saúde) e mesch na base e dados Pubmed da National Library of Medicine, tendo como critério de inclusão estudos primários, disponíveis em sua totalidade, publicados nos últimos 6 anos, em qualquer idioma que atendessem a temática do estudo. Resultados: Analisou-se 6 artigos que abordaram a avaliação clínica no primeiro período do trabalho de parto, na qual observou-se que a avalição neste período do trabalho de parto é essencial para o acompanhamento da evolução do parto. Durante a avaliação é possível identificar a presença de alterações que podem levam a intercorrências na hora do parto, desta forma é fundamento a avaliação continua tanto do feto quanto da parturiente. Considerações Finais: A equipe deve prestar o cuidado utilizando técnicas, procedimentos e conhecimentos empregados nas diferentes fases do parto.

Palavras chaves: Parto, Trabalho de parto, Tocologia.

\section{SUMMARY}

Objective: To list in the literature scientific evidence about the importance of clinical evaluation in the first period of labor. Methodology: This is an integrative review of the literature, in which descriptors based on VHL (Virtual Health Library) and mesch based on PubMed data from the National Library of Medicine were consulted, with the primary inclusion criteria, available in their entirety, published in the last 6 years, in any language that would attend the study topic. Results: We analyzed six articles that addressed the clinical evaluation in the first period of labor, in which it was observed that the evaluation in this period of labor is essential for monitoring the evolution of labor. During the evaluation it is possible to identify the presence of alterations that can lead to complications at the time of childbirth, in this way it is fundamental the continuous evaluation of both the fetus and the parturient. Final considerations: The team should provide care using techniques, procedures and knowledge employed in the different stages of childbirth.

Key words: Childbirth, Labor, Tocology.

\footnotetext{
${ }^{1}$ Docente do Instituto Superior Múltiplo de Ensino, Teresina-PI.

*E-mail: oliveira.naza@gmail.com

${ }^{2}$ Instituto de Ensino Superior Múltiplo (IESM). Teresina-PI.

${ }_{3}^{3}$ Universidade Estadual do Piauí e Centro Universitário Santo Agostinho, Teresina-PI.
} 


\section{RESUMEN}

Objetivo: El contenido de la literatura científica científica acerca de la importancia de la clínica clínica en el período de trabajo. Metodología: Trata-se dé uma revisión integrativa de la literatura, en el cual se publicó el libro BVS (Biblioteca Virtual en Biblioteca) y la Biblioteca y la Biblioteca Nacional de Medicina, disponible en su totalidad, publicados los últimos 6 años, en la misma lengua que atendessem a temática do estudo. Resultado: Análisis de los 6 artículos que abordarán un avalúo clínico en el primer período de tiempo en el parto, en el cual se observará un avalúo en el período de trabajo y en el papel esencial para el acompañamiento y la evolución del mismo. Durante un avalúo es posible identificar una presencia de las alteraciones que se pueden realizar a través de una entrevista en la hora del parto, la forma de un avalúo continuo y no sólo el feto quanto da parturiente. Consideraciones finales: Equipo para el cuidado o el cuidado que utiliza técnicas, procedimientos y principios de las diferentes fases del parto.

Palavras chaves: Parto, Trabalho de parto, Tocologia.

\section{INTRODUÇÃO}

O Trabalho de Parto (TP) compreende o conjunto de fenômenos fisiológicos que conduz a dilatação do colo uterino, a progressão do feto através do canal de parto e a sua expulsão para o exterior. O parto natural no seu contexto natural e fisiológico compreende a ausência de medicações, adequação das intervenções que proporcionam a mulher vivenciar o parto da forma mais natural possível em que a liberação dos hormônios acontece dentro da fisiologia do processo (HENRIQUE et al. 2016).

Diversas substâncias são produzidas durante a gestação e no trabalho de parto, fazendo parte da fisiologia do nascimento. Durante o parto, o cérebro é, juntamente com o útero, o órgão mais ativo do corpo da mãe, pois é quem controla todo o delicado sistema hormonal que entra em ação para, em nível físico, comportamental e emocional, preparar a mulher para a maternidade (LEAL et al. 2014).

O trabalho de parto tem início com contrações uterinas fracas e com pouca frequência, com intervalos de 10 a 30 minutos entre cada contração. As contrações tornam-se mais frequentes e dolorosas quando 0 intervalo entre elas se aproxima de 2 a 3 minutos entre cada contração (NASCIMENTO et al. 2017).

O trabalho de parto é dividido em 4 períodos que compreende em dilatação, expulsivo, dequitação e Greenberg. O primeiro período que compreende o período de dilatação divide-se em duas fases. Na fase latente a fase inicial do trabalho de parto e mais lenta deste período, e culmina com a dilatação do colo até os $3 \mathrm{~cm}$ (RAMOS et al. 2018).

A fase latente tem início com as contrações uterinas regulares e estende-se até o início da fase ativa. É essencial durante esta fase a realização da assistência de enfermagem individualizada, realização de orientações e técnicas de respiração e relaxamento (MELO et al. 2014). Durante a primeira fase da dilatação (fase latente), é importante orientar a parturiente a ficar em posição vertical (de pé, caminhando ou sentada) ou em decúbito lateral, estas posições possibilitam maior intensidade e maior eficiência das contrações (LIMA et al. 2017).

É fundamental durante a assistência de enfermagem no trabalho de parto a avaliação contínua tanto do feto quanto da parturiente, é necessário a avaliação da dinâmica uterina, monitoramento dos batimentos cardiofetais, sinais vitais, monitoramento de perda de líquido amniótico, sangue, diminuição dos batimentos fetais e sinais de desconfortos apresentados pela parturiente (NASCIMENTO et al. 2017).

É importante que a enfermeira obstétrica realize a avaliação da parturiente e seja capaz de identificar possíveis complicação durante o trabalho de parto, visto que esses profissionais devem possuir competências para manejar tanto a gestação, o trabalho de parto, o parto e o nascimento normais quanto às situações de risco ou de complicações das mulheres e ou dos recém-nascidos (LEAL et al. 2014; APOLINÁRIO et al. 2016). 
Pretende-se com este estudo elencar na literatura evidências cientifica acerca da importância da avaliação clínica no período de dilatação do trabalho de parto.

\section{METODOLOGIA}

Trata-se de uma revisão integrativa da literatura, um dos recursos da prática baseada em evidência, que resume o passado da literatura empírica ou teórica, para fornecer uma compreensão mais abrangente de um fenômeno particular (ARAGÃO, 2011).

Sua elaboração inclui: definição do objetivo; estabelecimento de critérios de inclusão e exclusão para a seleção da amostra; definição das informações a serem extraídas dos artigos selecionados; análise, e discussão dos resultados. Para orientar este estudo, formulou-se a seguinte questão: "Quais as evidencias cientifica publicadas sobre a avaliação clínica no primeiro período do trabalho de parto?".

A busca foi realizada nas bases de dados por meio de descritores na base de dado BVS (Biblioteca Virtual em saúde) e mesch na base de dado Pubmed da National Library of Medicine, e por meio da combinação de descritores e palavras chaves nas mesmas bases (Quadro 1). Em todos os bancos de dados, foram utilizados termos em Português, Inglês e Espanhol.

Os critérios de inclusão definidos foram: estudos primários, disponíveis em sua totalidade, publicados nos últimos 6 anos, em qualquer idioma que atendessem a temática do estudo.

Foram excluídos da busca inicial capítulos de livros, resumos, textos incompletos, teses de doutorado, dissertações de mestrados e relatos técnicos. A pesquisa ocorreu em abril de 2018 por um pesquisador.

Utilizaram-se os seguintes bancos com seus respectivos descritores, como monstra no quadro 1 :

Quadro 1 - Elementos da estratégia PICO e descritores utilizados, 2018.

\begin{tabular}{|c|c|c|}
\hline ELEMENTOS & MESH & DECS \\
\hline P & & "Parto" \\
& "Parto" & "Parturition" \\
& & "Parto" \\
\hline I & & "Trabalho de Parto" \\
"Trabalho de & "NursingCare" & "Labor, Obstetric" \\
Parto" & & "Trabajo de Parto" \\
\hline Co & & "Tocologia" \\
"Tocologia" & "Qualityof Life" & "Midwifery" \\
& & 'Tocología" \\
\hline
\end{tabular}

Fonte: Descritores, Títulos e Palavras-chaves.

Os termos utilizados durante a pesquisa foram classificados e combinados nos bancos de dados, resultando em estratégias específicas de cada base, como mostra o quadro 2. 
Quadro 2 - Estratégias de busca utilizadas nas bases de dados BVS e PUBMED- TERESINA, PI, 2018.

\begin{tabular}{|c|c|c|c|c|}
\hline BASE DE DADOS & \multicolumn{1}{|c|}{ ESTRATÉGIA DE BUSCA } & RESULTADOS & FILTRADOS & SELECIONADOS \\
\hline $\begin{array}{c}\text { BVS } \\
\text { (descritores Decs) }\end{array}$ & $\begin{array}{l}\text { tw:(+id:("bde-32254" OR "biblio-837821" } \\
\text { OR "bde-26472" OR "bde-24498" OR "lil- } \\
666761 " \text { OR "lil-697573")) AND } \\
\text { (instance:"regional") }\end{array}$ & 3.430 & 432 & 6 \\
\hline $\begin{array}{c}\text { PubMed } \\
\text { (descriptorsMeSH) }\end{array}$ & $\begin{array}{l}((((\text { Parturition) OR Parturition)) AND } \\
\text { ((Labor, Obstetric) OR Labor, Obstetric)) } \\
\text { AND ((Midwifery) OR Midwifery) }\end{array}$ & 1.936 & 397 & 0 \\
\hline
\end{tabular}

Fonte: Bases de dados.

Figura 1 - Fluxo do processo de seleção dos estudos para a revisão integrativa, 2018. (n=6).

Questão norteadora: Quais evidencias cientifica acerca da importância da avaliação no primeiro período clinico do trabalho de parto?.

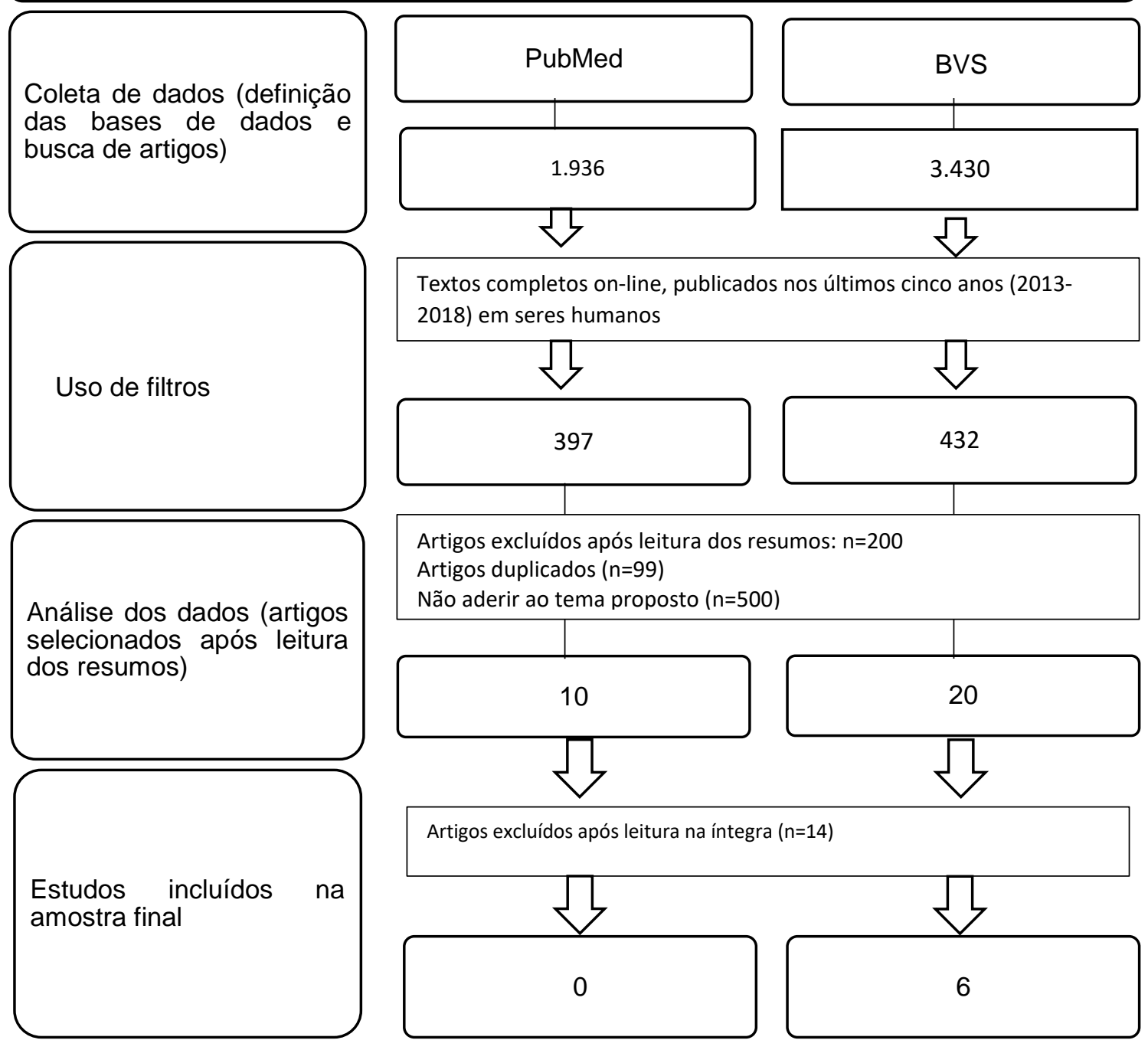




\section{RESULTADO}

A maioria dos estudos teve uma abordagem quantitativa (4/66\%); e houve a prevalência de série de casos $(04 / 66 \%)$. Todos os artigos (100\%) estavam no idioma português, a maioria dos artigos apresentaram nível de evidência concentrado em dois (02/33\%) e cinco (3/50\%). Todos os artigos (100\%) obtiveram grau de recomendação A. Todos os estudos foram realizados no Brasil (100\%) e a maioria das publicações foram concentradas no ano de 2013 (3/50\%), como mostra na tabela 1.

Tabela 1 - Análise descritiva das produções científicas acerca da atuação da equipe de enfermagem na sala de vacina e suas condições de funcionamento. Teresina, PI 2018. $(\mathrm{N}=6)$

\begin{tabular}{|c|c|c|}
\hline VARIÁVEIS & $\mathbf{N}$ & $\%$ \\
\hline \multicolumn{3}{|l|}{ Abordagem do estudo } \\
\hline Quantitativo & 04 & 66 \\
\hline Revisão integrativa & 02 & 33 \\
\hline \multicolumn{3}{|c|}{ Delineamento da pesquisa } \\
\hline Revisão sistemática & 02 & 33 \\
\hline Série de Casos & 04 & 66 \\
\hline \multicolumn{3}{|l|}{ Idioma } \\
\hline Português & 06 & 100 \\
\hline \multicolumn{3}{|c|}{ Classificação da evidência } \\
\hline Um & 01 & 16 \\
\hline Dois & 02 & 33 \\
\hline Cinco & 03 & 50 \\
\hline \multicolumn{3}{|l|}{ Grau de Recomendação } \\
\hline A & 06 & 100 \\
\hline \multicolumn{3}{|l|}{ Procedência } \\
\hline Brasil & 06 & 100 \\
\hline \multicolumn{3}{|l|}{ Distribuição temporal } \\
\hline 2013 & 03 & 50 \\
\hline 2014 & 01 & 16 \\
\hline 2016 & 01 & 16 \\
\hline 2018 & 01 & 16 \\
\hline
\end{tabular}

Houve uma grande variação no perfil amostral em todos os artigos. Em sua maioria os estudos avaliaram avaliação clínica no primeiro período do trabalho de parto, como mostra o quadro 3. 
Quadro 3 - Distribuição das publicações incluídas segundo os autores, delineamento da pesquisa, objetivo principal, amostra, principais resultados, nível de evidência e grau de recomendação. Teresina, $\mathrm{PI}, 2018$.

\begin{tabular}{|c|c|c|c|c|c|c|}
\hline Autor & $\begin{array}{l}\text { Delineamento da } \\
\text { pesquisa }\end{array}$ & Objetivo principal & Amostral & Principais resultados & NE & GR \\
\hline $\begin{array}{l}\text { BARBIERI, M. } \\
\text { et al. } 2013\end{array}$ & $\begin{array}{l}\text { Estudo clínico } \\
\text { experimental } \\
\text { randomizado }\end{array}$ & $\begin{array}{l}\text { Avaliar de forma isolada e combinada a } \\
\text { utilização do banho quente de aspersão e } \\
\text { exercícios perineais realizados com bola suíça } \\
\text { durante o trabalho de parto e a percepção da } \\
\text { dor. }\end{array}$ & \begin{tabular}{|l|}
15 \\
parturientes
\end{tabular} & $\begin{array}{l}\text { Quando as intervenções em estudo foram associadas a } \\
\text { diminuição da dor foi significativa. Não houve diferença } \\
\text { significativa no escore de dor, quando as intervenções } \\
\text { foram isoladas. }\end{array}$ & 2 & A \\
\hline $\begin{array}{l}\text { HENRIQUE, } \\
\text { A. J. et al. } \\
2016\end{array}$ & $\begin{array}{l}\text { Ensaio clínico } \\
\text { randomizado }\end{array}$ & $\begin{array}{l}\text { Conhecer a influência do banho quente e } \\
\text { exercício perineal com bola suíça, de forma } \\
\text { isolada e combinada, sobre a progressão do } \\
\text { trabalho de parto. }\end{array}$ & \begin{tabular}{|l|}
44 \\
parturientes
\end{tabular} & $\begin{array}{l}\text { A pesquisa mostrou aumento estatisticamente significante } \\
\text { na frequência da contração uterina com uso isolado } \\
\text { ( } p=0,025) \text { e associado da bola suíça }(p<0,001) \text {, um } \\
\text { aumento também significante na frequência cardíaca fetal } \\
\text { com uso isolado e associado do banho quente }(p<0,001)\end{array}$ & 2 & A \\
\hline $\begin{array}{l}\text { MAZONI, S.R. } \\
\text { et al. } 2013\end{array}$ & $\begin{array}{l}\text { Estudo } \\
\text { observacional }\end{array}$ & $\begin{array}{l}\text { Identificar a presença dos indicadores clínicos } \\
\text { da dor em situação de parto e correlacionar a } \\
\text { referência verbal de intensidade de dor com a } \\
\text { ocorrência de contrações uterinas, como } \\
\text { proposta de validação clínica do diagnóstico } \\
\text { de enfermagem dor de parto }\end{array}$ & \begin{tabular}{|l|}
55 \\
parturientes
\end{tabular} & $\begin{array}{l}\text { Testaram-se } 22 \text { características definidoras, } 6 \text { presentes } \\
\text { na maioria das participantes nas duas fases: relato verbal } \\
\text { ou codificado, evidência observada de contração uterina, } \\
\text { alteração do tônus muscular, evidência observada de dor, } \\
\text { comportamento expressivo e expressão facial de dor. }\end{array}$ & 5 & $A$ \\
\hline $\begin{array}{l}\text { OSÓRIO, S. } \\
\text { M. B. et al. } \\
2014\end{array}$ & $\begin{array}{c}\text { Revisão } \\
\text { Sistemática }\end{array}$ & $\begin{array}{l}\text { Avaliar a efetividade de métodos não } \\
\text { farmacológicos no alívio da dor durante o } \\
\text { trabalho de parto. }\end{array}$ & $\begin{array}{ll}\text { Bases de } \\
\text { dados }\end{array}$ & $\begin{array}{l}\text { Evidenciou-se que a massagem, a aromaterapia, o banho } \\
\text { de imersão, a acupuntura e a acupressão são eficazes } \\
\text { métodos para aliviar a dor no trabalho de parto, pois além } \\
\text { de diminuírem a percepção dolorosa, ainda reduzem os } \\
\text { níveis de ansiedade e de estresse. }\end{array}$ & 1 & $B$ \\
\hline $\begin{array}{l}\text { PIESZAK, G. } \\
\text { M. et al. } 2013\end{array}$ & $\begin{array}{l}\text { Pesquisa descritiva, } \\
\text { exploratória }\end{array}$ & $\begin{array}{l}\text { Compreender como a equipe de enfermagem } \\
\text { percebe o cuidar no processo de parturição. }\end{array}$ & \begin{tabular}{|l|}
10 \\
profissionais
\end{tabular} & $\begin{array}{l}\text { Concluiu-se que a humanização da assistência ainda } \\
\text { representa um desafio para estes profissionais, porém } \\
\text { percebem-se avanços neste sentido. }\end{array}$ & 5 & A \\
\hline $\begin{array}{l}\text { RAMOS W. M. } \\
\text { A. et al. } 2018\end{array}$ & Estudo documental & $\begin{array}{l}\text { Identificar as boas práticas desenvolvidas pela } \\
\text { Enfermeira Obstétrica em uma Maternidade } \\
\text { Municipal do Rio de Janeiro e analisar a } \\
\text { assistência das Enfermeiras Obstétricas nas } \\
\text { Boas Práticas no momento do parto. }\end{array}$ & \begin{tabular}{ll}
\multicolumn{2}{l}{ Maternidade } \\
Municipal da \\
Cidade do \\
Rio & de \\
Janeiro &
\end{tabular} & $\begin{array}{l}\text { Foi constatado que as Enfermeiras Obstétricas } \\
\text { ofereceram assistência à } 367 \text { parturientes utilizando as } \\
\text { boas práticas de forma segura. O estudo demonstrou que } \\
\text { o uso das boas práticas tem grande influência na } \\
\text { recuperação da fisiologia materna, na adaptação do } \\
\text { recém-nascido e redução da anemia na primeira infância }\end{array}$ & 5 & A \\
\hline
\end{tabular}

Fonte: Elaboração própria.

$\mathrm{NE}^{*}$ Numero de Evidência

$\mathrm{GR}^{*}$ Grau de Recomendação

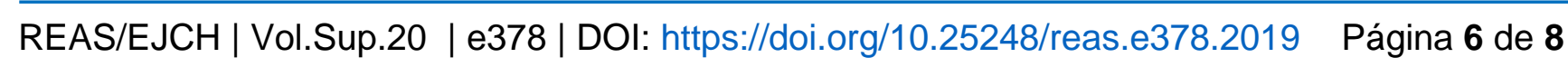




\section{DISCUSSÃO}

Segundo a literatura durante o trabalho de parto é indispensável planejar e implementar estratégias que possam ajudar as mulheres a vivenciar experiências de parto com medo e ansiedade mínimos, ao lançar mão de mecanismos de enfrentamento na redução dos índices de insatisfação e ajudá-las a recuperar o controle durante o parto (RAMOS et al., 2018).

Durante a fase latente é necessário realizar a avaliar dinâmica uterina registrando sua frequência, duração e intensidade, monitorar o batimento cardíaco fetal (BCF) antes, durante e após as contrações para identificar a presença de desacelerações intrapélvicas e registrar no prontuário (NASCIMENTO et al., 2017).

Quando às alterações no BCF e/ou nas contrações uterinas é necessário informar ao médico, deve-se ainda monitorar perda de líquido, sangramento uterino, contrações eficientes e diminuição dos movimentos fetais (MELO et al., 2014).

No primeiro estágio do trabalho de parto o exame de toque vaginal deve ser realizado, no máximo, a cada duas ou mais horas. A quantidade e o momento de realizar o exame vaginal deve ser considerado, com critério, para permitir a avaliação adequada do progresso do trabalho de parto (NASCIMENTO et al., 2017).

$O$ enfermeiro obstetra exerce todas as atividades de enfermagem, cabendo privativamente, como integrante da equipe de saúde, a assistência à parturiente, o acompanhamento da evolução do parto e execução do parto normal sem qualquer problema, tanto de origem materna quanto fetal, que dificulte ou impeça o parto, sendo permitida a episiotomia e a episiorrafia, no qual esse profissional está amparado por lei para exercer suas atividades com eficiência e segurança (SANTANA et al., 2016).

Estudo realizado por Apolinário et al., (2016) identificou que existe práticas realizadas na assistência ao parto e nascimento que devem ser estimuladas e outras eliminadas. Entre as práticas que devem ser estimuladas, destaca-se: o cuidado com a privacidade da mulher; a participação do acompanhante de escolha durante todo o processo, a utilização de métodos não farmacológicos de alívio da dor no trabalho de parto o incentivo do contato pele- a-pele da mãe e do recém-nascido e amamentação logo após o nascimento.

No entanto, foram encontradas práticas que devem ser utilizadas com cautela ou eliminadas da assistência ao parto e que foram mencionadas pelas puérperas como: a transferência da mulher durante 0 período expulsivo para a sala de parto, a posição de litotomia no momento do nascimento, o jejum no trabalho de parto, punção venosa de rotina, toques vaginais em curto espaço de tempo e por mais de um profissional (APOLINÁRIO et al., 2016).

$\mathrm{Na}$ fase latente por ser a fase mais lenta do trabalho de parto, é fundamental que oriente a parturiente a realizar exercícios respiratórios, uso de bola e a deambulação. A dor relatada pela parturiente deve ser respeitada de acordo com seus limites, visto que para a maioria das mulheres, o parto é sinônimo de dor (MELO et al., 2014).

Estudos evidenciam que a massagem, a aromaterapia, o banho de imersão, a acupuntura e a acupressão são eficazes métodos para aliviar a dor no trabalho de parto, pois além de diminuírem a percepção dolorosa, ainda reduzem os níveis de ansiedade e de estresse principalmente quando aplicada na primeira fase do trabalho de parto (OSÓRIO et al., 2014; BARBIERI et al., 2013).

Os benefícios advindos do uso da bola suíça estão relacionados a posição vertical favorecer e pelo alinhamento do eixo fetal com a pelve materna, bem como a descida e progressão fetal no canal de parto, em conjunto com o relaxamento produzido pelo exercício muscular do períneo (HENRIQUE et al., 2016).

A finalidade do posicionamento e movimentação pélvica durante o trabalho de parto estão relacionados com a diminuição do desconforto materno, ajudando na circulação uterina e aumento da intensidade das contrações, assim como na diminuição da duração do tempo do trabalho de parto (MAZONI et al., 2013).

Observa-se que é necessário incentiva a participação ativa das mulheres na hora do parto oferecendo encorajamento e estímulo durante todo processo. Para isso é preciso que haja a promoção de uma 
comunicação efetiva com a parturiente, que lhe proporcione conforto, Ihe amenize a sensação de medo e angústia e transmita segurança e confiança (RAMOS et al., 2018).

A confiança permite que o trabalho em equipe seja facilitado, pois, quando a relação está pautada na confiança e segurança previamente estabelecidas entre o profissional e a parturiente, é possível realizar um parto envolto em uma esfera de cuidado e tranquilidade (PIESZAK et al., 2013; MAZONI et al., 2013).

É importante que os profissionais da enfermagem mantenham com a parturiente uma relação de ajuda, e adote atitudes que fortaleça as habilidades da parturiente para que essa possa atuar de forma eficaz e satisfatória durante o trabalho de parto. É importante que este profissional execute suas atividades junto ao acompanhante e parturiente, informando-os sobre a evolução e condutas a serem realizadas durante o processo de nascimento (LEAL et al., 2014). É necessário que o acompanhante tenha conhecimento de toda assistência prestada a parturiente e colabore junto com a equipe na prática de exercícios e redução da ansiedade da parturiente.

\section{CONSIDERAÇÕES FINAIS}

Este estudo permitiu discutir a importância da avaliação da parturiente no primeiro período clínico do trabalho do parto, segundo a literatura científica. Concluiu-se então, que a avaliação no primeiro período clínico do trabalho de parto deve ser realizada de forma contínua tanto do feto quanto da parturiente. A assistência prestada ao parto está ligada a mudanças nas práticas e rotinas institucionais, e inserida no contexto da humanização do parto e nascimento. A equipe deve prestar o cuidado utilizando técnicas, procedimentos e conhecimentos empregados nas diferentes fases do parto.

Ressalta-se como limitação do estudo a existência de poucos artigos específicos sobre a avaliação no primeiro período clinico do trabalho de parto, que impossibilitou maiores comparações e aprofundamentos na temática. Diante do fato faz-se necessário a realização de novos estudos, com vistas a ampliar a produção de evidências científicas que subsidiem a avalição da parturiente no primeiro período clinico do trabalho de parto.

\section{REFERÊNCIAS}

1. HENRIQUE AJ, GABRIELLONI MC, CAVALCANTI ACV et al. Hidroterapia e bola suíça no trabalho de parto: ensaio clínico randomizado. Acta Paul Enferm. v.29, n.6, p:686-92, 2016.

2. LEAL MC, PEREIRA APE, DOMINGUES RMSM et al. Intervenções obstétricas durante o trabalho de parto e parto em mulheres brasileiras de risco habitual. Cad. Saúde Pública, Rio de Janeiro, v.30, p:17-47, 2014.

3. NASCIMENTO ACA, LIMA ALP, ARAÚJO JC et al. Assistência de enfermagem na fase latente do trabalho de parto: Relato de experiência. international nursing congress. p:9-12, 2017.

4. RAMOS WMA, AGUIAR BGC, CONRAD D et al. Contribuição da enfermeira obstétrica nas boas práticas da assistência ao parto e nascimento. J. res.: fundam. care. v.10, n.1, p:173-179, 2018.

5. MELO AS, SANTOS AG, NERY IS et al. Diagnósticos de enfermagem na saúde da mulher: parturientes na primeira fase do trabalho de parto. Rev enferm UFPE, Recife, v.8, n.6, p:1467-73, jun., 2014.

6. LIMAI MFG, PEQUENO AMC, RODRIGUES DP et al. Desenvolvendo competências no ensino em enfermagem obstétrica: aproximações entre teoria e prática. Rev Bras Enferm. v.70, n.5, p:1110-6, 2017.

7. APOLINÁRIO D, RABELO M, WOLFF LDG et al. Práticas na atenção ao parto e nascimento sob a perspectiva das puérperas. Rev Rene. v.17, n.1, p:20-8, 2016.

8. ARAGÃO, J. Introdução aos estudos quantitativos utilizados em pesquisas científicas. Rev. Práxis, v.3, n.6, 2011.

9. RAMOS WMA, AGUIAR BGC, CONRAD D et al. Contribuição da enfermeira obstétrica nas boas práticas da as- sistência ao parto e nascimento. Rev Fund Care, v.10, n.1, p:173-179, 2018.

10. SANTANA ACS, MENEZES NGA, SOUZA DS. Atuação e importância do enfermeiro obstetra na atenção ao parto natural. Anais 2016: 18

11. OSÓRIO SMB, JÚNIOR LGS, NICOLAU AIO. Avaliação da efetividade de métodos não farmacológicos no alívio da dor do parto. Rev Rene. v.15, n1, p:174-84, 201.

12. BARBIERI M, HENRIQUE AJ, CHORS FM et al. Banho quente de aspersão, exercícios perineais com bola suíça e dor no trabalho de parto. Acta Paul Enferm. v.26, n.5, p:478-84, 2013.

13. HENRIQUE AJ, GABRIELLONI MC, CAVALCANTI ACV et al. Hidroterapia e bola suíça no trabalho de parto: ensaio clínico randomizado. Acta Paul Enferm. v.29, n.6, p:686-92, 2016.

14. MAZONI SR, CARVALHO EC, SANTOS CB. Validação clínica do diagnóstico de enfermagem dor de parto. Rev. Latino-Am. Enfermagem, v.21, n.09, p: 155-29, 2013.

15. PIESZAK GM, TERRA MG, NEVES ET al. Percepção dos profissionais de enfermagem acerca do cuidar em centro obstétrico. Rev Rene. v.14, n.3, p:568-78, 2013. 